\title{
Erratum to: Methods of harmonic synthesis for global geopotential models and their first-, second- and third-order gradients
}

\author{
E. Fantino $^{1} \cdot$ S. Casotto ${ }^{1,2}$
}

Published online: 5 October 2015

(C) Springer-Verlag Berlin Heidelberg 2015

\section{Erratum to: J Geod (2009) 83:595-619 \\ DOI 10.1007/s00190-008-0275-0}

The expression for the coefficient $A_{m}^{(r r)}$ given in the third row of the first column of Table 5 should be corrected to include the factor $\bar{C}_{n m}$ as follows:

$$
A_{m}^{(r r)}=\sum_{n=m}^{N}\left(\frac{a}{r}\right)^{n}(n+1)(n+2) \bar{C}_{n m} \bar{P}_{n}^{m} .
$$

Similarly, the expression for the coefficient $A_{m}^{(r r r)}$ given in the fourth row of the first column of Table 6 should be corrected to include the factor $\bar{C}_{n m}$ as follows:

$$
A_{m}^{(r r r)}=\sum_{n=m}^{N}\left(\frac{a}{r}\right)^{n}(n+1)(n+2)(n+3) \bar{C}_{n m} \bar{P}_{n}^{m} .
$$

The right-hand sides of the formulas appearing in Table 10 should be corrected to include the factor $(1 / r)$ as follows:

$a_{1}=\frac{1}{r} \sum_{m=1}^{N} m\left(A_{m}^{(1)} c_{m-1}+B_{m}^{(1)} s_{m-1}\right) \cos ^{m-1} \phi$
$a_{2}=\frac{1}{r} \sum_{m=1}^{N} m\left(B_{m}^{(1)} c_{m-1}-A_{m}^{(1)} s_{m-1}\right) \cos ^{m-1} \phi$

The online version of the original article can be found under doi:10.1007/s00190-008-0275-0.

S. Casotto

stefano.casotto@unipd.it

1 Dipartimento di Astronomia, Università di Padova, Vicolo dell'Osservatorio 3, 35122 Padua, Italy

2 Center for Space Studies (CISAS) "G. Colombo", Università di Padova, Via Venezia 15, 35131 Padua, Italy $a_{3}=\frac{1}{r} \sum_{m=0}^{N}\left(A_{m}^{(2)} c_{m}+B_{m}^{(2)} s_{m}\right) \cos ^{m} \phi$

$a_{4}=-\frac{1}{r} \sum_{m=0}^{N}\left(A_{m}^{(3)} c_{m}+B_{m}^{(3)} s_{m}\right) \cos ^{m} \phi$.

The right-hand sides of the formulas appearing in Table 11 should be corrected to include the factor $(1 / r)^{2}$. The expression of the coefficient $a_{11}$ contains further misprints. Hence, the formulas of Table 11 should read:

$a_{11}=\frac{1}{r^{2}} \sum_{m=2}^{N} m(m-1)\left(A_{m}^{(1)} c_{m-2}+B_{m}^{(1)} s_{m-2}\right) \cos ^{m-2} \phi$

$a_{12}=\frac{1}{r^{2}} \sum_{m=2}^{N} m(m-1)\left(B_{m}^{(1)} c_{m-2}-A_{m}^{(1)} s_{m-2}\right) \cos ^{m-2} \phi$

$a_{13}=\frac{1}{r^{2}} \sum_{m=1}^{N} m\left(A_{m}^{(2)} c_{m-1}+B_{m}^{(2)} s_{m-1}\right) \cos ^{m-1} \phi$

$a_{14}=-\frac{1}{r^{2}} \sum_{m=1}^{N} m\left(A_{m}^{(3)} c_{m-1}+B_{m}^{(3)} s_{m-1}\right) \cos ^{m-1} \phi$

$a_{22}=-a_{11}$

$a_{23}=\frac{1}{r^{2}} \sum_{m=1}^{N} m\left(B_{m}^{(2)} c_{m-1}-A_{m}^{(2)} s_{m-1}\right) \cos ^{m-1} \phi$

$a_{24}=-\frac{1}{r^{2}} \sum_{m=1}^{N} m\left(B_{m}^{(3)} c_{m-1}-A_{m}^{(3)} s_{m-1}\right) \cos ^{m-1} \phi$

$a_{33}=\frac{1}{r^{2}} \sum_{m=0}^{N}\left(A_{m}^{(4)} c_{m}+B_{m}^{(4)} s_{m}\right) \cos ^{m} \phi$

$a_{34}=-\frac{1}{r^{2}} \sum_{m=0}^{N}\left(A_{m}^{(5)} c_{m}+B_{m}^{(5)} s_{m}\right) \cos ^{m} \phi$ 
$a_{44}=\frac{1}{r^{2}} \sum_{m=0}^{N}\left(A_{m}^{(6)} c_{m}+B_{m}^{(6)} s_{m}\right) \cos ^{m} \phi$.

The right-hand sides of the formulas appearing in Table 12 should be corrected by including the factor $(1 / r)^{3}$. The expressions of the coefficients $a_{111}, a_{143}, a_{222}$ and $a_{444}$ contain further misprints. Hence, the formulas of Table 12 should read:

$$
\begin{aligned}
& a_{111}=\frac{1}{r^{3}} \sum_{m=3}^{N} m(m-1)(m-2) \\
& \times\left(A_{m}^{(1)} c_{m-3}+B_{m}^{(1)} s_{m-3}\right) \cos ^{m-3} \phi \\
& a_{112}=-a_{222} \\
& a_{113}=\frac{1}{r^{3}} \sum_{m=2}^{N} m(m-1)\left(A_{m}^{(2)} c_{m-2}+B_{m}^{(2)} s_{m-2}\right) \cos ^{m-2} \phi \\
& a_{114}=-\frac{1}{r^{3}} \sum_{m=2}^{N} m(m-1)\left(A_{m}^{(3)} c_{m-2}+B_{m}^{(3)} s_{m-2}\right) \cos ^{m-2} \phi \\
& a_{123}=\frac{1}{r^{3}} \sum_{m=2}^{N} m(m-1)\left(B_{m}^{(2)} c_{m-2}-A_{m}^{(2)} s_{m-2}\right) \cos ^{m-2} \phi \\
& a_{124}=-\frac{1}{r^{3}} \sum_{m=2}^{N} m(m-1)\left(B_{m}^{(3)} c_{m-2}-A_{m}^{(3)} s_{m-2}\right) \cos ^{m-2} \phi \\
& a_{143}=-\frac{1}{r^{3}} \sum_{m=1}^{N} m\left(A_{m}^{(5)} c_{m-1}+B_{m}^{(5)} s_{m-1}\right) \cos ^{m-1} \phi \\
& a_{221}=-a_{111} \\
& a_{222}=-\frac{1}{r^{3}} \sum_{m=3}^{N} m(m-1)(m-2) \\
& \times\left(B_{m}^{(1)} c_{m-3}-A_{m}^{(1)} s_{m-3}\right) \cos ^{m-3} \phi \\
& a_{223}=-a_{113} \\
& a_{224}=-a_{114} \\
& a_{243}=-\frac{1}{r^{3}} \sum_{m=1}^{N} m\left(B_{m}^{(5)} c_{m-1}-A_{m}^{(5)} s_{m-1}\right) \cos ^{m-1} \phi \\
& a_{331}=\frac{1}{r^{3}} \sum_{m=1}^{N} m\left(A_{m}^{(4)} c_{m-1}+B_{m}^{(4)} s_{m-1}\right) \cos ^{m-1} \phi \\
& a_{332}=\frac{1}{r^{3}} \sum_{m=1}^{N} m\left(B_{m}^{(4)} c_{m-1}-A_{m}^{(4)} s_{m-1}\right) \cos ^{m-1} \phi \\
& a_{333}=\frac{1}{r^{3}} \sum_{m=0}^{N}\left(A_{m}^{(7)} c_{m}+B_{m}^{(7)} s_{m}\right) \cos ^{m} \phi \\
& a_{334}=-\frac{1}{r^{3}} \sum_{m=0}^{N}\left(A_{m}^{(8)} c_{m}+B_{m}^{(8)} s_{m}\right) \cos ^{m} \phi
\end{aligned}
$$

$$
\begin{aligned}
& a_{441}=\frac{1}{r^{3}} \sum_{m=1}^{N} m\left(A_{m}^{(6)} c_{m-1}+B_{m}^{(6)} s_{m-1}\right) \cos ^{m-1} \phi \\
& a_{442}=\frac{1}{r^{3}} \sum_{m=1}^{N} m\left(B_{m}^{(6)} c_{m-1}-A_{m}^{(6)} s_{m-1}\right) \cos ^{m-1} \phi \\
& a_{443}=\frac{1}{r^{3}} \sum_{m=0}^{N}\left(A_{m}^{(9)} c_{m}+B_{m}^{(9)} s_{m}\right) \cos ^{m} \phi \\
& a_{444}=-\frac{1}{r^{3}} \sum_{m=0}^{N}\left(A_{m}^{(10)} c_{m}+B_{m}^{(10)} s_{m}\right) \cos ^{m} \phi .
\end{aligned}
$$

The expression for the coefficient $A_{m}^{(2)}$ in Table 13 should read:

$A_{m}^{(2)}=\sum_{n=m}^{N} \rho_{n} \bar{C}_{n m} \mathrm{~d} \bar{H}_{n}^{m} / \mathrm{d} u$

There are missing terms in the expression for the function $\bar{W}_{n}^{m}$ in Table 14 . The formula should read:

$$
\begin{aligned}
\bar{W}_{n}^{m}= & (n+m+1)(n+m+2)(n+m+3) \bar{H}_{n}^{m} \\
& +3 u(n+m+2)(n+m+3) \mathrm{d} \bar{H}_{n}^{m} / \mathrm{d} u \\
& +3 u^{2} \mathrm{~d}^{2} \bar{H}_{n}^{m} / \mathrm{d} u^{2} \\
& +u^{3} \mathrm{~d}^{3} \bar{H}_{n}^{m} / \mathrm{d} u^{3} .
\end{aligned}
$$

The letter $l$ in Eqs. (76) and (77) should be replaced by the letter $r$, so that the two equations read:

$$
\begin{aligned}
V_{n m} & =\frac{(-1)^{n}}{(n-m) !}\left(\frac{\partial}{\partial x}+\mathrm{i} \frac{\partial}{\partial y}\right)^{m}\left(\frac{\partial}{\partial z}\right)^{(n-m)}\left(\frac{1}{r}\right), \\
V_{n n} & =(-1)^{n}\left(\frac{\partial}{\partial x}+\mathrm{i} \frac{\partial}{\partial y}\right)^{n}\left(\frac{1}{r}\right),
\end{aligned}
$$

respectively.

A + sign should be replaced by a - sign in Eq. (94), in the following way:

$$
\begin{aligned}
\bar{S}_{n m}^{(\alpha \beta \gamma)}= & \frac{(-1)^{\beta / 2}}{(-2)^{\alpha+\beta}} \sum_{p=0}^{\alpha} \sum_{q=0}^{\beta}\left[\epsilon^{+} A^{+} \bar{S}_{n-\eta, m+\sigma}\right. \\
& \left.-\left(1-\delta_{0 m}\right) \epsilon^{-} A^{-} \bar{S}_{n-\eta,-m+\sigma}\right] .
\end{aligned}
$$

Finally, the term $3 \eta$ appearing in the upper limits of the summations in Eqs. (101), (102) and (103) and related text should be replaced by $\eta$ :

$$
\frac{\partial^{\eta} V}{\partial x^{\alpha} \partial y^{\beta} \partial z^{\gamma}}=\frac{G M}{a^{1+\eta}} \sum_{m=0}^{N+\eta}\left(A_{m}^{(\alpha \beta \gamma)} \cos m \lambda+B_{m}^{(\alpha \beta \gamma)} \sin m \lambda\right),
$$




$$
\begin{aligned}
A_{m}^{(\alpha \beta \gamma)} & =\sum_{n=m}^{N+\eta} \bar{C}_{n m}^{(\alpha \beta \gamma)} \bar{E}_{n m}, \\
B_{m}^{(\alpha \beta \gamma)} & =\sum_{n=m}^{N+\eta} \bar{S}_{n m}^{(\alpha \beta \gamma)} \bar{E}_{n m} .
\end{aligned}
$$

Acknowledgments The authors wish to thank David F. Crouse for detecting the missing $1 / r$ factors and Fan Gao for further detecting the missing $1 / r^{2}$ factors and the misprints in Eqs. (94) and (101)-(103). 